\title{
Recoil-ion trapping for precision mass measurements
}

\author{
A. Herlert ${ }^{1, a}$, S. Van Gorp ${ }^{2}$, D. Beck ${ }^{3}$, K. Blaum ${ }^{4}$, M. Breitenfeldt ${ }^{5, b}$, R.B. Cakirli6,c ${ }^{6}$ S. George ${ }^{4, d}$, U. Hager ${ }^{7, e}$, \\ F. Herfurth ${ }^{3}$, A. Kellerbauer ${ }^{4}$, D. Lunney ${ }^{8}$, R. Savreux ${ }^{3}$, L. Schweikhard ${ }^{5}$, and C. Yazidjian ${ }^{3}$ \\ 1 CERN, Physics Department, 1211 Geneva 23, Switzerland \\ 2 K.U. Leuven, Instituut voor Kern- en Stralingsfysica, Celestijnenlaan 200 D, 3001 Leuven, Belgium \\ 3 GSI Helmholtzzentrum für Schwerionenforschung GmbH, Planckstr. 1, 64291 Darmstadt, Germany \\ 4 Max-Planck-Institut für Kernphysik, Saupfercheckweg 1, 69117 Heidelberg, Germany \\ 5 Ernst-Moritz-Arndt-Universität, Institut für Physik, 17487 Greifswald, Germany \\ 6 Istanbul University, Department of Physics, 34134 Istanbul, Turkey \\ 7 University of Jyväskylä, Department of Physics, P.O. Box 35 (YFL), 40014 Jyväskylä, Finland \\ 8 CSNSM-IN2P3-CNRS, Université de Paris Sud, 91405 Orsay, France
}

Received: 22 March 2012 / Revised: 6 June 2012

Published online: 26 July 2012 - (C) Società Italiana di Fisica / Springer-Verlag 2012

Communicated by N. Alamanos

\begin{abstract}
For the first time masses of recoiling daughter ions have been measured that were held after beta-decay in a buffer-gas-filled Penning trap. From the masses of the trapped beta-decaying manganese ions ${ }^{61-63} \mathrm{Mn}^{+}$and the daughter recoil-ions ${ }^{61-63} \mathrm{Fe}^{+}$the $Q$ values of ${ }^{61-63} \mathrm{Mn}$ have been deduced with absolute uncertainties of about $5 \mathrm{keV}$. The observed yields of iron ions are compared to the results from simulations, which confirm a recoil-ion trapping efficiency of about $50 \%$.
\end{abstract}

\section{Introduction}

The region of neutron-rich nuclei around $N=40$ has been the subject of many investigations with the aim of shedding light on the question how nuclear structure evolves between the major neutron shells $N=28$ and 50. Indeed, data, e.g., on neutron-rich chromium $[1,2]$, vanadium [3] and iron isotopes [4-6] at $N=40$ have shown a variation of the deformation and an increase of the collectivity near $N=40$ [7]. The onset of collectivity is seen as an indication supporting a new island of inversion [8] in analogy to the one known at $N=20$, e.g., for ${ }^{32} \mathrm{Mg}$ [9]. Mass measurements on neutron-rich nickel, copper and gallium isotopes showed no indication of a sub-shell behavior [10]. Nevertheless, data across $N=40$ were still missing, e.g., for neutron-rich manganese and iron isotopes, which would give further information on the mass surface in that region.

\footnotetext{
a Present address: FAIR GmbH, Planckstr. 1, 64291 Darmstadt, Germany, e-mail: alexander.herlert@fair-center.eu

b Present address: K.U. Leuven, Instituut voor Kern- en Stralingsfysica, Celestijnenlaan 200 D, 3001 Leuven, Belgium.

c Present address: Max-Planck-Institut für Kernphysik, Saupfercheckweg 1, 69117 Heidelberg, Germany.

d Present address: Ernst-Moritz-Arndt-Universität, Institut für Physik, 17487 Greifswald, Germany.

e Present address: Colorado School of Mines, Golden, CO 80401, USA.
}

Penning trap mass spectrometry is presently the method of choice for high-precision mass determinations on stable as well as short-lived nuclei [11-19]. Numerous such measurements have been performed at the ISOLDE facility [20] at CERN by use of the dual Penning trap mass spectrometer ISOLTRAP [21]. ISOLTRAP has access to a large variety of radioactive ion beams available at ISOLDE. However, although more than 900 nuclides can be delivered with yields sufficiently high for mass measurements at ISOLTRAP, the production process with protons impinging on thick targets and subsequent diffusion and ionization leads to gaps in the chart of nuclides: Due to their chemical and physical properties, the isotopes of certain elements are not released from the target and are therefore not available. A particular example are the iron isotopes which are required for the study of nuclear structure in the region around $N=40$.

In order to circumvent the limits of radioisotope production, Penning traps can be employed, which allow for the storage of ions for extended times without significant losses. This feature can be used to produce nuclides by transmutation, e.g., nuclear $\beta$ decay in the trap volume: The short-lived ions are stored until they eventually decay. The large axial trapping potential, the magnetic field, and the damping effect of the buffer gas are enough to retain the recoiling daughter-nuclide ions in the trap volume. The feasibility of this approach has been shown in a preliminary study with ${ }^{37} \mathrm{~K}^{+}$ions which $\beta^{+}$decayed into ${ }^{37} \mathrm{Ar}^{+}$ in the trap [22]. In the present work, this procedure was 
Table 1. Time structure of the experimental steps in the ion traps for standard operation and for in-trap decay.

\begin{tabular}{|c|c|c|c|c|}
\hline \multirow[t]{2}{*}{ Procedure } & \multicolumn{2}{|c|}{ Standard operation } & \multicolumn{2}{|c|}{ In-trap decay } \\
\hline & Duration (ms) & Element & Duration (ms) & Element \\
\hline \multicolumn{5}{|c|}{ RFQ buncher and cooler } \\
\hline Accumulation & $0.05-100$ & Mn & $0.05-100$ & $\mathrm{Mn}$ \\
\hline Cooling & 8 & Mn & 8 & $\mathrm{Mn}$ \\
\hline \multicolumn{5}{|c|}{ Preparation Penning trap } \\
\hline Axial cooling & 150 & $\mathrm{Mn}, \mathrm{Fe}$ & 1000 & $\mathrm{Mn}, \mathrm{Fe}$ \\
\hline Magnetron rf excitation & 5 & $\mathrm{Mn}, \mathrm{Fe}$ & 5 & $\mathrm{Mn}, \mathrm{Fe}$ \\
\hline Quadrupolar rf excitation & 100 & Mn & 100 & $\mathrm{Fe}$ \\
\hline Cooling of cyclotron motion & 100 & $\mathrm{Mn}$ & 100 & $\mathrm{Fe}$ \\
\hline \multicolumn{5}{|c|}{ Precision Penning trap } \\
\hline Magnetron rf excitation & 10 & Mn & 10 & $\mathrm{Fe}$ \\
\hline Dipolar rf excitation (cleaning) & 50 & Mn & 50 & $\mathrm{Fe}$ \\
\hline Quadrupolar rf excitation & $100-1200$ & $\mathrm{Mn}$ & $100-1200$ & $\mathrm{Fe}$ \\
\hline
\end{tabular}

applied to the decay of neutron-rich manganese isotopes into iron isotopes in order to gain access to these nuclides for mass measurements for the first time at ISOLDE. In addition, the observed iron yields are compared to $a b$ initio simulations to provide more quantitative information on the efficiency of the recoil-ion trapping in a buffer-gasfilled Penning trap.

\section{Experimental setup and procedure}

The neutron-rich manganese ions studied in this work were produced by impinging $1.4 \mathrm{GeV}$ protons from the Proton Synchrotron Booster at CERN on a uraniumcarbide target equipped with a tungsten cavity for selective laser ionization [23]. The ISOLDE ion source was operated at $30 \mathrm{kV}$, and isobars were separated with the general purpose mass separator GPS and afterwards sent to the ISOLTRAP setup.

The ISOLTRAP mass spectrometer is described in detail in [21]. It consists of a radiofrequency quadrupole buncher and cooler (RFQ) [24] to prepare the 30 to $60 \mathrm{keV}$ quasi-continuous beam from ISOLDE for subsequent trapping, and two Penning traps for further mass purification and high-precision mass measurements. In the first Penning trap [25] the ions of interest are selected by use of mass-specific buffer-gas cooling [26]. As part of the ion cooling process, the ions are initially kept in the trap for $150 \mathrm{~ms}$ to axially center them before application of the rf excitations. For the in-trap-decay scheme this duration is increased to one second in order to allow the stored manganese ions to decay in the trap. The most important experimental steps and their durations are given in table 1.

The method of recoil-ion trapping has already been described in detail for the case of $\beta^{+}$decay [22]. In contrast, the presently studied manganese isotopes decay by $\beta^{-}$ emission, i.e. the daughter nuclide will be doubly charged
Table 2. Half-life, spin and parity of ground and isomeric states of manganese isotopes. Data taken from [27]. Note that for ${ }^{62} \mathrm{Mn}$ the assignment of the ground and isomeric state is not known (the energy difference between ground-state and excited-state isomer is estimated as $\Delta E=0(150) \mathrm{keV}$ as deduced from systematic trends [28]).

\begin{tabular}{|cccc|}
\hline Isotope & $\Delta E(\mathrm{keV})$ & Half-life & $J^{\pi}$ \\
\hline \hline${ }^{56} \mathrm{Mn}$ & & $2.58 \mathrm{~h}$ & $3^{+}$ \\
${ }^{57} \mathrm{Mn}$ & & $85.4 \mathrm{~s}$ & $5 / 2^{-}$ \\
${ }^{58} \mathrm{Mn}$ & & $3.0 \mathrm{~s}$ & $1^{+}$ \\
${ }^{58 m} \mathrm{Mn}$ & 71.78 & $65.4 \mathrm{~s}$ & $(4)^{+}$ \\
${ }^{59} \mathrm{Mn}$ & & $4.59 \mathrm{~s}$ & $(5 / 2)^{-}$ \\
${ }^{60} \mathrm{Mn}$ & & $280 \mathrm{~ms}$ & $1^{+}$ \\
${ }^{60 m} \mathrm{Mn}$ & 271.90 & $1.77 \mathrm{~s}$ & $4^{+}$ \\
${ }^{61} \mathrm{Mn}$ & & $670 \mathrm{~ms}$ & $(5 / 2)^{-}$ \\
${ }^{62 / 62 m} \mathrm{Mn}$ & & $671 \mathrm{~ms}$ & $\left(4^{+}\right)$ \\
${ }^{62 / 62 m} \mathrm{Mn}$ & & $92 \mathrm{~ms}$ & $\left(1^{+}\right)$ \\
${ }^{63} \mathrm{Mn}$ & & $275 \mathrm{~ms}$ & $\left(5 / 2^{-}\right)$ \\
\hline
\end{tabular}

immediately after the decay rather than being neutral (neglecting additional electron shake-off). For the present studies, only the singly charged iron ions were selected by tuning to the proper cyclotron frequency, i.e., those ions that underwent charge-exchange reactions in the buffergas environment as has been observed, e.g., in the case of doubly charged xenon ions [29].

Since neither the masses of the neutron-rich manganese isotopes nor those of the iron isotopes are well known, both are of interest and can be accessed in one experimental run using the in-trap-decay method. In order to have sufficient decays of the parent ions on the experimental time scale (half-lives below one second, see table 2), only the iron isotopes ${ }^{61-63} \mathrm{Fe}$ have been studied. 


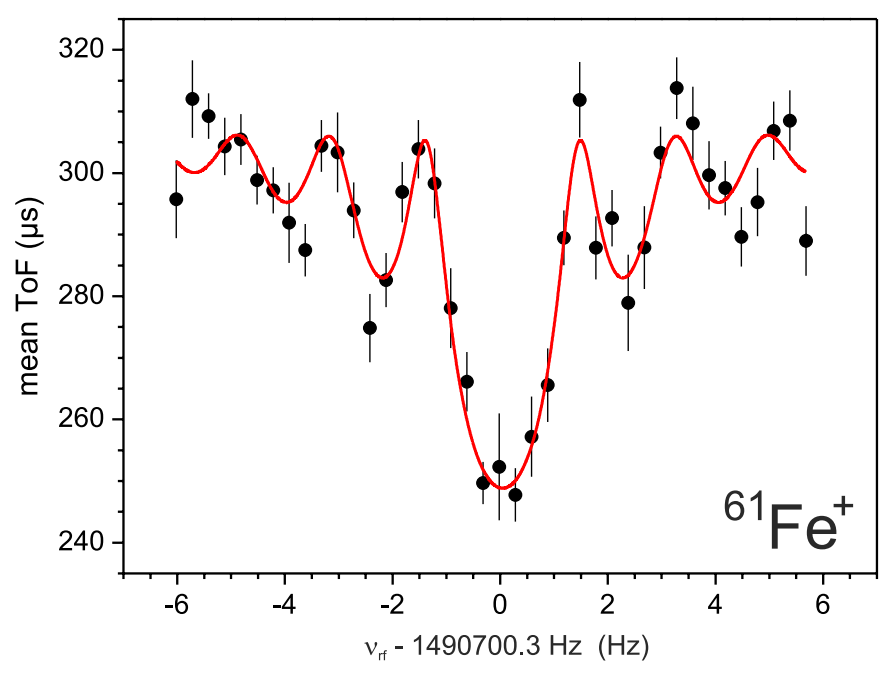

Fig. 1. (Color online) Ion-cyclotron resonance of ${ }^{61} \mathrm{Fe}^{+}$ions. The solid line is a fit of the theoretical line shape [30] to the data points.

\section{Results}

\subsection{Mass determination}

The mass of an ion is determined via the ratio of its cyclotron frequency in a Penning trap to that of a reference ion with well-known mass. The cyclotron frequencies

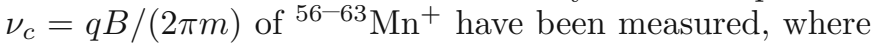
$B$ is the magnetic field strength in the Penning trap and $m$ and $q$ are the mass and charge of the stored ion. After recoil-ion trapping the ${ }^{61-63} \mathrm{Fe}^{+}$ions were transferred to the precision Penning trap where their cyclotron frequencies were determined. As an example, a cyclotron resonance taken for ${ }^{61} \mathrm{Fe}^{+}$is shown in fig. 1 .

A summary of all measured frequency ratios $r=\nu_{c, r e f} /$ $\nu_{c}$ is given in table 3 . The atomic mass $m$ of the nuclide of interest can be derived by

$$
m=r\left(m_{r e f}-m_{e}\right)+m_{e},
$$

with the atomic mass $m_{\text {ref }}$ of the reference nuclide and the electron mass $m_{e}$. By convention not the atomic mass but rather the mass excess $M E$ is listed in the AtomicMass Evaluation (AME) [31,32], which is defined as the difference $M E=m-A$ between the atomic mass (in atomic mass units) and the mass number $A$, taking the conversion factor $1 u=931494.0090(71) \mathrm{keV}$ [31].

Cyclotron frequencies of the stable isotopes ${ }^{41} \mathrm{~K}$ and ${ }^{85} \mathrm{Rb}$ as well as of ${ }^{56,57} \mathrm{Mn}$ were taken as cross-checks. For ${ }^{85} \mathrm{Rb}$ a deviation by more than two standard deviations from the literature mass value was obtained, which is probably due to a non-optimal injection of ions much heavier than the reference ions ${ }^{39} \mathrm{~K}^{+}$into the precision trap. Although no deviation for the mass of the stable ${ }^{41} \mathrm{~K}$ isotope was observed, all uncertainties for the frequency ratios given in table 3 were modified by adding in quadrature $6 \times 10^{-8}$ as an additional systematic uncertainty. This brings the measured mass excess of ${ }^{85} \mathrm{Rb}$ within one standard deviation of the literature value. Otherwise, the analysis procedure followed the one described in [33]. Despite the additional systematic uncertainty, the mass excess values obtained have total uncertainties below $4 \mathrm{keV}$ except in the case of ${ }^{63} \mathrm{Fe}$, for which only one resonance was recorded, resulting in a larger statistical uncertainty.

\subsection{Identification of isomers}

In some cases long-lived isomers can be more strongly populated in the production process than the ground state. All such states studied in this work are given in table 2. Usually, with proton-induced fission, the high-spin isomeric state is more strongly populated than the ground state (see, e.g., $[34,35]$ or [36] for far asymmetric fission). In the case of ${ }^{62} \mathrm{Mn}$ the spin assignment of the isomers is not known and therefore a careful analysis is needed.

As an example, the cyclotron resonance of ${ }^{58} \mathrm{Mn}^{+}$is plotted in fig. 2 for two different rf excitation durations. While for a duration of $T_{r f}=300 \mathrm{~ms}$ the two states are not resolved, their presence can be inferred by comparing single- and double-resonance fits. For $T_{r f}=1200 \mathrm{~ms}$ the cyclotron resonance shows the presence of the ground state very clearly (see fig. 2(bottom)). This state has a much lower abundance as compared to the isomeric state at lower cyclotron frequency, i.e., at higher mass. Thus, as only a few ions are present in the trap in each experimental cycle, the amount of contaminating ground-state ions is negligible and there is no significant influence on the mass determination of the isomeric state.

While in the case of ${ }^{58} \mathrm{Mn}$ both states were observed and thus their relative abundance is known, the situation for ${ }^{60} \mathrm{Mn}$ and ${ }^{62} \mathrm{Mn}$ is more complicated. In both cases no trace of a second state was observed in the time-of-flight (ToF) spectra or cyclotron resonances. Especially in the case of ${ }^{62} \mathrm{Mn}$ the excitation energy of the isomer is not known and therefore no suitable excitation duration can be chosen in order to achieve separation. Furthermore, in both cases the high-spin state is most probably more strongly populated and delivered by ISOLDE, independent of the respective half-lives and resulting losses due to $\beta$ decay.

In order to obtain additional information to help identify the ground or isomeric state, the data were analyzed with respect to the absolute number of ions in the precision trap as a function of storage time. Note that the decay loss of the parent ions can only be monitored by measuring the number of remaining parent ions and not at the number of appearing daughter ions. The latter mostly leave the trap due to the shallow trapping potential.

As an example, the absolute number of ions per cycle in the precision trap is plotted as a function of the cyclotron rf excitation duration for ${ }^{62} \mathrm{Mn}^{+}$in fig. 3(center), which is the main contribution to the storage time in the precision trap. The ion signal was integrated over the expected ToF range of parent ions only (taking into account the shorter ToF due to in-resonance quadrupolar rf excitation, see, e.g., fig. 3(top) for ${ }^{63} \mathrm{Mn}^{+}$). Since the experimental time was limited, only a few different excitation durations were applied. The resulting values for the half-lives are shown 
Table 3. Frequency ratios measured between ${ }^{58 m, 59,60 m, 61-63} \mathrm{Mn}$ and ${ }^{61-63} \mathrm{Fe}$ and the reference ion ${ }^{39} \mathrm{~K}^{+}$(mass of ${ }^{39} \mathrm{~K} m=$ $39.96399848(21) u[32]$ with $1 u=931494.0090(71) \mathrm{keV}[31]$ and $\left.m_{e}=548579.9110(12) \times 10^{-9} u\right)$. Frequency ratios of stable alkali ions as well as long-lived manganese ions ${ }^{56,57} \mathrm{Mn}^{+}$relative to ${ }^{39} \mathrm{~K}^{+}$were measured as cross-checks. An additional systematic uncertainty of $6 \times 10^{-8}$ has been added in quadrature to the uncertainty of the frequency ratios (for details see text). In addition to the data measured in this work, frequency ratios from an earlier campaign (in 2003) are listed, for which only mass excess values have been published so far.

\begin{tabular}{|c|c|c|c|}
\hline Ion & $r$ & $M E_{\text {exp }}(\mathrm{keV})$ & $M E_{l i t}(\mathrm{keV})[31]$ \\
\hline \multicolumn{4}{|c|}{ Data from 2006 (as published in [37]) } \\
\hline${ }^{58 m} \mathrm{Mn}^{+}$ & $1.4870354024(735)$ & $-55755.4(2.7)$ & $-55840(30)$ \\
\hline${ }^{59} \mathrm{Mn}^{+}$ & $1.5127070218(631)$ & $-55525.0(2.3)$ & $-55480(30)$ \\
\hline${ }^{60 m} \mathrm{Mn}^{+}$ & $1.5384502463(648)$ & $-52695.8(2.4)$ & $-52910(90)$ \\
\hline${ }^{61} \mathrm{Mn}^{+}$ & $1.5641418011(653)$ & $-51741.8(2.4)$ & $-51560(230)$ \\
\hline${ }^{62 / 62 m} \mathrm{Mn}^{+}$ & $1.5899051939(718)$ & $-48180.6(2.6)$ & $-48040(220)$ \\
\hline${ }^{63} \mathrm{Mn}^{+}$ & $1.615606112(102)$ & $-46886.8(3.7)$ & $-46350(260)$ \\
\hline${ }^{61} \mathrm{Fe}^{+}$ & $1.5639440154(729)$ & $-58920.2(2.7)$ & $-58921(20)$ \\
\hline${ }^{62} \mathrm{Fe}^{+}$ & $1.5896104560(773)$ & $-58877.8(2.8)$ & $-58901(14)$ \\
\hline${ }^{63} \mathrm{Fe}^{+}$ & $1.615365034(155)$ & $-55636.5(5.6)$ & $-55550(170)$ \\
\hline \multicolumn{4}{|c|}{ Cross-check data from 2006 (as published in [37]) } \\
\hline${ }^{56} \mathrm{Mn}^{+}$ & $1.4356730300(696)$ & $-56910.7(2.5)$ & $-56909.7(0.7)$ \\
\hline${ }^{57} \mathrm{Mn}^{+}$ & $1.4613225062(714)$ & $-57484.0(2.6)$ & $-57486.8(1.8)$ \\
\hline${ }^{41} \mathrm{~K}^{+}$ & $1.0512822800(609)$ & $-35558.6(2.2)$ & $-35559.07(0.19)$ \\
\hline${ }^{85} \mathrm{Rb}^{+}$ & $2.1792700552(686)$ & $-82165.0(2.5)$ & $-82167.331(0.011)$ \\
\hline \multicolumn{4}{|c|}{ ISOLTRAP data from $2003^{(a)}$} \\
\hline${ }^{56} \mathrm{Mn}^{+}$ & $0.6587862217(182)$ & $-56910.3(1.4)$ & $-56909.7(0.7)$ \\
\hline${ }^{57} \mathrm{Mn}^{+}$ & $0.6705559418(275)$ & $-57486.4(2.2)$ & $-57486.8(1.8)$ \\
\hline
\end{tabular}

(a) Data from [38] taken with reference ion ${ }^{85} \mathrm{Rb}^{+}$. Only the mass excess was published in [39]. These data were included in the AME2003 mass evaluation [31].

in fig. 3(bottom) together with the literature values for the ground and isomeric states.

For ${ }^{60} \mathrm{Mn}$ the half-life of the ground state is $0.280 \mathrm{~s}$ [40] while the excited-state isomer has a half-life of $1.77 \mathrm{~s}$. In the case of ${ }^{62} \mathrm{Mn}$ a short-lived isomeric state was identified by Gaudefroy et al. [41] which has a half-life of $92 \mathrm{~ms}$. An assignment of the ground state was, however, not possible and therefore it is not clear if the high- or the low-spin state is the ground state. It is assumed in the following that the longer-lived state is the ground state.

In all cases the half-lives determined are about a factor of two smaller than the literature value of the longer-lived state. Additional loss mechanisms cannot be excluded. Furthermore, fluctuations in the ISOLDE ion production may lead to systematic deviations in the measured absolute ion numbers. Therefore, the half-lives obtained from the decay loss in the measurement Penning trap are not sufficient for unambiguous identification.

\subsection{Ion yields}

As an additional check, the yield observed at ISOLTRAP can be compared to the manganese yield as delivered by ISOLDE using a resonance-ionization laser ion source (RILIS) [42]. The measurement cycle had a fixed duration as shown in table 1 and therefore the measured yield can be corrected for decay losses during the storage in the various ion traps. The result is shown in fig. 4 , where the filled circles are the corrected yields of manganese ions from the RFQ buncher and cooler and the empty diamonds indicate the ISOLDE yields as tabulated in the yield data base (data from [42]) scaled by the stopping and trapping efficiency of $0.5 \%$ of the ISOLTRAP RFQ. The efficiency of $0.5 \%$ was determined by use of a stableion beam from ISOLDE and application of the ISOLDE beam gate for production of well-defined number of ions entering the RFQ. The empty squares denote yield data taken during the experimental run at the ISOLDE tapestation system (also scaled by $0.5 \%$ ). Up to mass number $A=59$ a lower yield was observed as compared with the expected (scaled) ISOLDE yield, which is most probably due to a detuned ion transport. In addition, the first data points (up to mass $A=59$ ) were recorded during beamsharing mode of the GPS mass separator, i.e., ISOLTRAP taking the central mass after the separator magnet and additional lower-mass beam was deflected to a separate user 

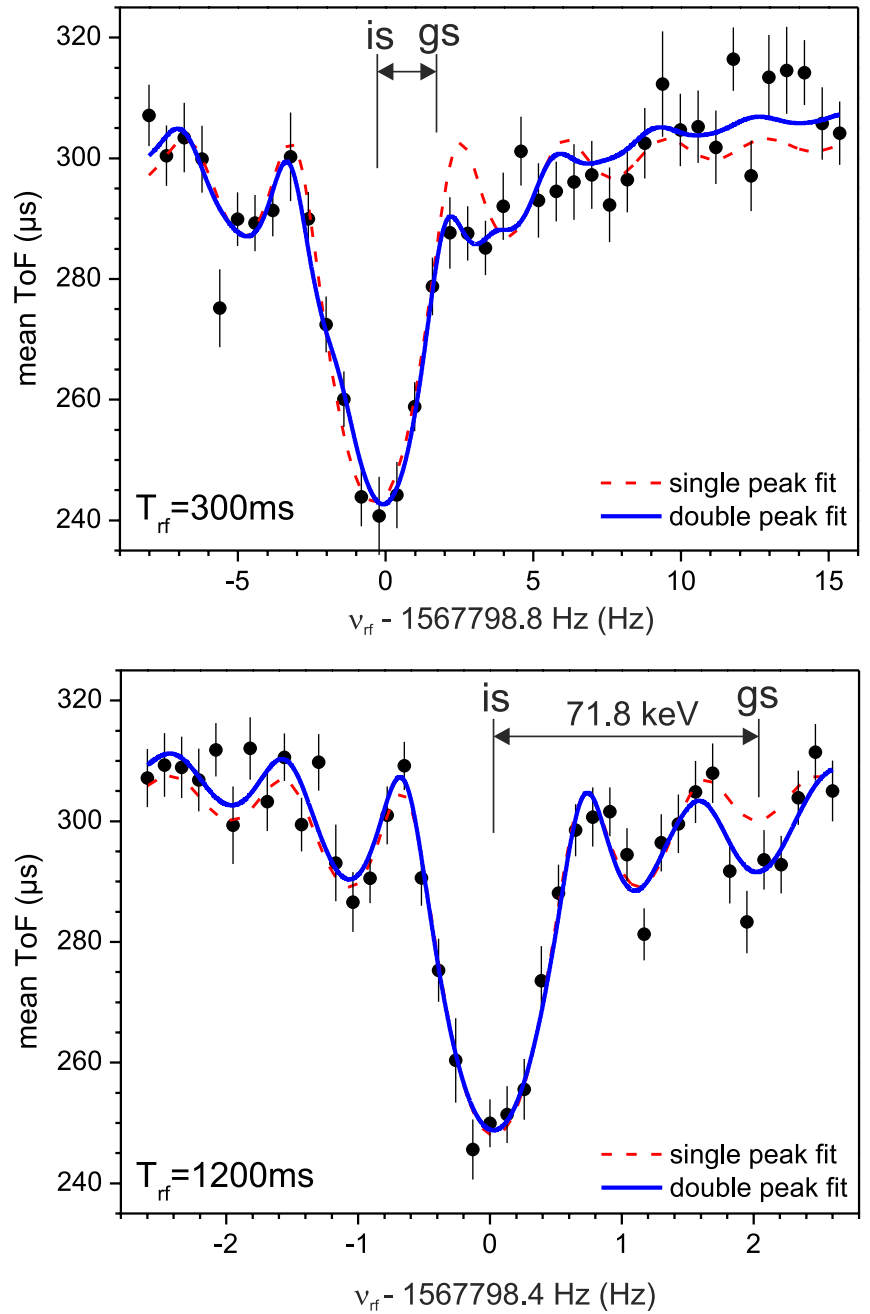

Fig. 2. (Color online) Ion cyclotron resonances of ${ }^{58} \mathrm{Mn}^{+}$for excitation durations $T_{r f}=300 \mathrm{~ms}$ (top) and $T_{r f}=1200 \mathrm{~ms}$ (bottom). The solid and dashed lines are fits of the line shape [30] to the data points. The dashed line shows a fit for a single resonance $\left(\chi_{\nu}^{2}=1.6\right.$ and 1.5 for $T_{r f}=300 \mathrm{~ms}$ and $T_{r f}=1200 \mathrm{~ms}$, respectively) while the solid line gives the result for two resonances within the frequency range $\left(\chi_{\nu}^{2}=1.0\right.$ and 1.1 for $T_{r f}=300 \mathrm{~ms}$ and $T_{r f}=1200 \mathrm{~ms}$, respectively). The ground state (gs) and isomeric state (is) are marked together with the energy difference. See text for details.

for collections. After retuning of the ion transport (measurements from $A=59$ to 63 ) and running as the only user, a satisfactory agreement of the measured ISOLDE yield with the yield at ISOLTRAP can be seen. For the more neutron-rich manganese isotopes the trend of the observed yield follows the expected yield data.

In the case of $A=60,62$ the observed yield was corrected according to the shorter half-life of the respective isomers (see table 2) and the resulting yield data are plotted as empty circles in fig. 4. Clearly, the yields are much higher than expected from the trend, especially in the case of ${ }^{62} \mathrm{Mn}$, which, together with the deduced half-life data, gives a strong indication that in the case of ${ }^{60} \mathrm{Mn}$ and ${ }^{62} \mathrm{Mn}$ the longer-lived states have been observed in the measurement trap, i.e. the isomeric state for ${ }^{60} \mathrm{Mn}$.
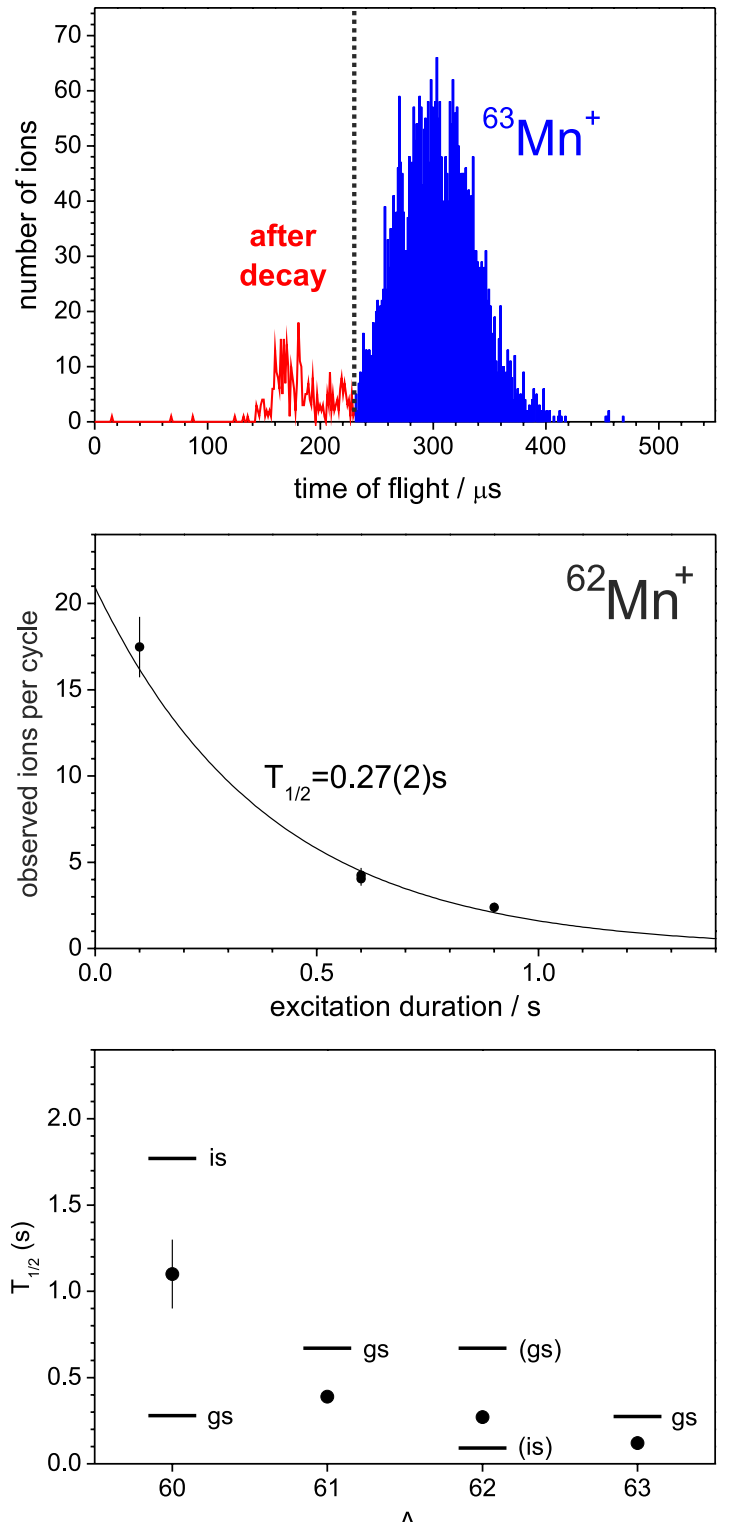

Fig. 3. (Color online) Top: ToF spectrum of ${ }^{63} \mathrm{Mn}^{+}$ions (accumulation of several experimental cycles) measured at the final detector after application of the quadrupolar rf excitation in the precision trap. The dashed line separates the signal from ${ }^{63} \mathrm{Mn}^{+}$ions from the signal of daughter ions (and/or ionized residual gas) after $\beta$ decay. Center: Number of ${ }^{62} \mathrm{Mn}^{+}$ions observed at the final ToF ion detector as a function of the duration of the quadrupolar rf excitation in the precision Penning trap. The solid line is a weighted fit of an exponential decay to the data points. Bottom: Half-lives of ${ }^{60-63} \mathrm{Mn}$ as deduced from exponential fits as shown in the top graph (filled circles). The expected half-lives of the ground state (gs) and isomeric state (is) are indicated by lines. In the case of ${ }^{62} \mathrm{Mn}$ the assignment to either the ground state or the isomeric state is not clear.

For an estimate of the recoil-ion-trapping efficiency, the initial yield of the precursor manganese ions is determined from the number of iron ions observed at the ToF ion detector by correcting for the in-trap decay in the preparation Penning trap as well as decay losses during 


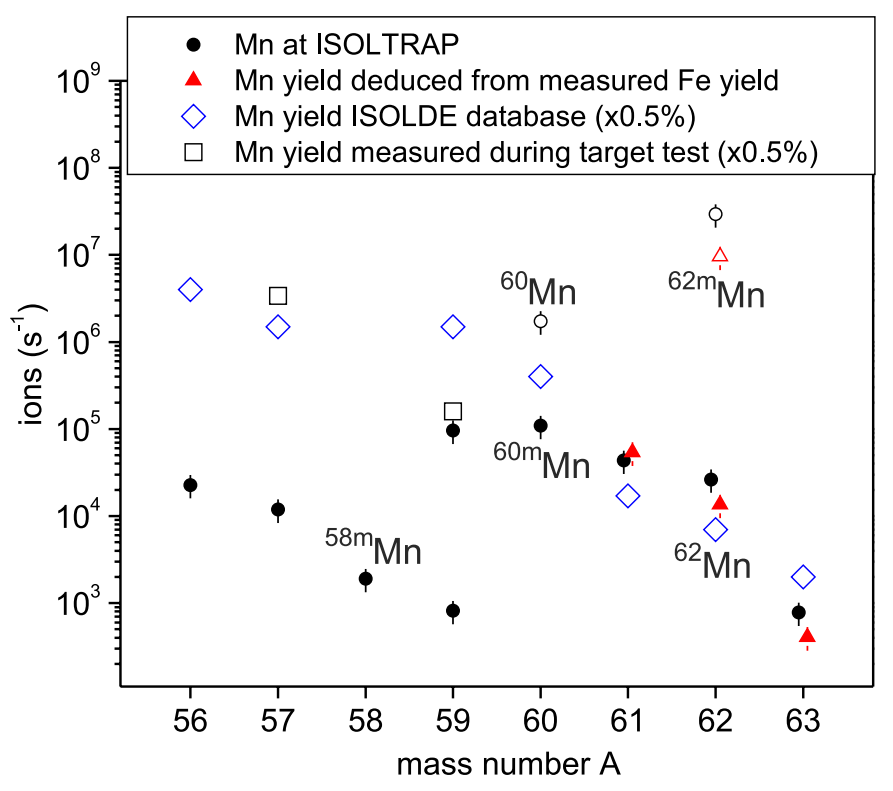

Fig. 4. (Color online) Yield of manganese ions as measured with the final ion detector at ISOLTRAP (filled circles). In addition, the yield of manganese ions as expected from the ISOLDE yield data base is shown (empty diamonds) together with data points measured in the beginning of the run as a target test (empty squares). A transport efficiency of $0.5 \%$ from the ISOLDE ion source to the ToF ion detector of ISOLTRAP is assumed to rescale the ISOLDE data. Data points denoted by filled triangles give the manganese yield as deduced from the observed iron yield measured at the ion detector. Data points denoted by empty circles and triangles show the deduced manganese yields if the shorter-lived isomers of ${ }^{60,62} \mathrm{Mn}$ are assumed.

the transport to the preparation trap. The resulting manganese yields are plotted in fig. 4 (filled triangles).

\section{Discussion}

\subsection{Q-values from mass measurements}

The mass excess values determined in this work are compared to the literature values as given in the 2003 AtomicMass Evaluation (AME2003) [31]. The filled and empty circles in fig. 5 denote the AME2003 data relative to the ISOLTRAP values for the ground and isomeric states, respectively. In general, there is good agreement for the ground-state data within the uncertainties of the literature values. The new data mainly reduce the uncertainties of the mass excess values.

For the manganese isotopes, a trend towards smaller mass excess values is observed when going to more neutron-rich isotopes. The literature value of ${ }^{63} \mathrm{Mn}$ is deviating by more than $500 \mathrm{keV}$, however, with an uncertainty of $260 \mathrm{keV}$. In the case of the isomeric states ${ }^{58 m} \mathrm{Mn}$ and ${ }^{60 m} \mathrm{Mn}$, a deviation by the amount of the excitation energy is observed, which possibly indicates an incorrect assignment of levels in the results from previous $\beta$-decay experiments that are included in the mass evaluation AME2003. As energy differences are used to deduce the mass of the
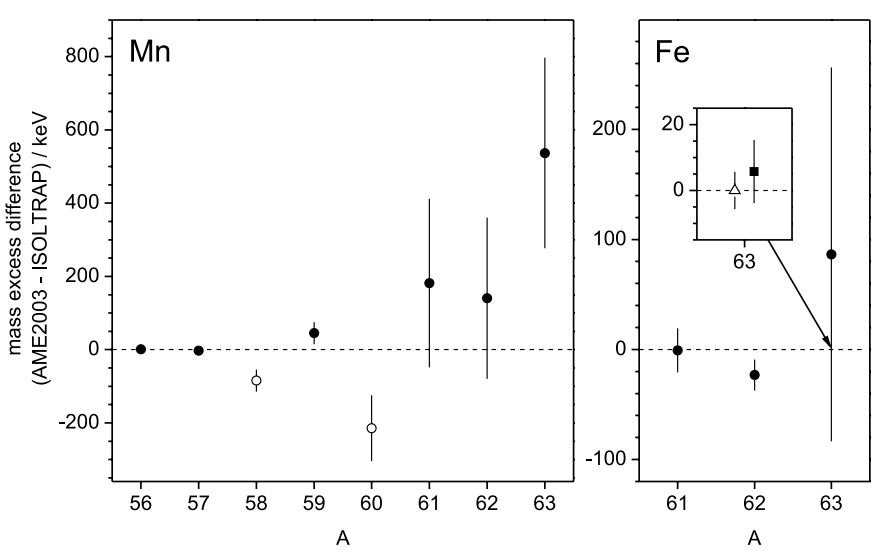

Fig. 5. Mass excess values from the Atomic-Mass Evaluation 2003 data [31] (filled and empty circles for ground and isomeric states, respectively) relative to the values for manganese and iron isotopes determined in this work (the error band is too narrow to be shown on this scale). The inset shows an additional recent result for ${ }^{63} \mathrm{Fe}$ from the LEBIT Penning trap experiment [43] (filled square) which is compared to the ISOLTRAP value (empty triangle).

respective parent or daughter nuclide, incorrect level assignments may lead to systematic shifts. A thorough investigation is pursued in a separate publication which includes in addition to the data presented in table 3 also new data on neutron-rich manganese isotopes up to ${ }^{66} \mathrm{Mn}$ and a full new mass evaluation [37]. The present work concentrates on the recoil-ion trapping and its efficiency.

In the case of the iron isotopes, a recent result [43] from the LEBIT Penning trap mass spectrometer has been added for comparison as well (see the inset of fig. 5, filled square). This shows the good agreement of the ISOLTRAP and LEBIT data for ${ }^{63} \mathrm{Fe}$, confirming the accuracy on the level of a few $\mathrm{keV}$ by independent experimental data.

Since precise mass excess values are available for three pairs of mother-daughter nuclei, measured in the same experiment under the same conditions, the $Q$-values of the manganese isotopes ${ }^{61-63} \mathrm{Mn}$ can be deduced as summarized in table 4 . These values will be used in the following simulations of the in-trap decay of the manganese ions and the recoil-ion trapping. Note that for the $Q$-values sub-keV precision can be achieved if a dedicated experimental cycle and measurement procedures are applied (see, e.g., [44-46] and references therein). To this end the cyclotron frequencies of the parent and daughter nuclide ions are measured alternately without using a reference-mass measurement. This will give a data set of a mass doublet with negligible systematic shifts. The $Q$-value is determined by the difference of the cyclotron frequencies and its uncertainty. This measurement scheme was not applied in this work but is in principle possible.

\subsection{Efficiency of recoil-ion trapping and simulations}

The observed yield of iron isotopes can be used as a measure of the recoil-ion trapping efficiency. As mentioned 
Table 4. $Q$-values as deduced from the experimental data and resulting maximum recoil energies (as calculated from eq. (8)) of the daughter nuclei.

\begin{tabular}{|cccc|}
\hline Nuclide & $Q_{\text {exp }}(\mathrm{keV})$ & $Q_{\text {lit }}(\mathrm{keV})[32]$ & $\begin{array}{c}\text { Max. recoil } \\
\text { energy }(\mathrm{eV})\end{array}$ \\
\hline \hline${ }^{61} \mathrm{Mn}$ & $7178(4)$ & $7370(230)$ & 518 \\
${ }^{62} \mathrm{Mn}$ & $10697(4)$ & $10860(220)$ & 1086 \\
${ }^{63} \mathrm{Mn}$ & $8750(7)$ & $9190(310)$ & 729 \\
\hline
\end{tabular}

Table 5. Ratio of experimental manganese yields and fraction of product ions trapped after in-trap decay of manganese isotopes in the Penning trap as simulated for two different charge states $z$ (see text for details). The branching ratios for the case of additional $\gamma$ decays are discussed in the text. The experimental values of the manganese yields with and without recoil-ion trapping are taken from fig. 4 to calculate the ratio.

\begin{tabular}{|cc|cc|}
\hline Parent ion & Product ion & $z=1$ & $z=2$ \\
\hline \hline \multicolumn{3}{|c|}{$\begin{array}{c}\text { Ratio of experimental manganese yield } \\
\text { with/without recoil-ion trapping }\end{array}$} \\
\hline \multicolumn{3}{|c|}{${ }^{61} \mathrm{Mn}^{+}$} & $1.22(52)$ \\
${ }^{62} \mathrm{Mn}^{+}$ & $0.51(22)$ \\
${ }^{63} \mathrm{Mn}^{+}$ & ${ }^{62} \mathrm{Fe}^{+}$ & $0.52(22)$ \\
\hline \hline \multicolumn{3}{|c|}{$\beta$ decay to gs $(\%)$} \\
\hline${ }^{63} \mathrm{Fe}^{+}$ & 52.6 \\
${ }^{61} \mathrm{Mn}^{+}$ & ${ }^{61} \mathrm{Fe}^{z+}$ & 74.6 \\
${ }^{62} \mathrm{Mn}^{+}$ & ${ }^{62} \mathrm{Fe}^{z+}$ & 35.0 \\
${ }^{63} \mathrm{Mn}^{+}$ & ${ }^{63} \mathrm{Fe}^{z+}$ & 43.6 \\
\hline \hline \multicolumn{3}{|c|}{$\beta$ decay and $\gamma$ decay to gs $(\%)$} \\
\hline${ }^{61} \mathrm{Mn}^{+}$ \\
${ }^{62} \mathrm{Mn}^{+}$ & 51.1 \\
${ }^{63} \mathrm{Mn}^{++}$ & 38.7 \\
\hline
\end{tabular}

above, the initial manganese yield can be deduced and compared to the yield as measured without recoil ion trapping. The ratios of the averaged calculated and observed manganese yields are given in table 5(top). In the case of ${ }^{61} \mathrm{Mn}$, the yields are about the same, while for ${ }^{62} \mathrm{Mn}$ and ${ }^{63} \mathrm{Mn}$ the ratio is smaller at around $50 \%$, most probably due to losses of the recoil ions after $\beta$ decay.

For a more quantitative approach, ab initio simulations have been performed using the SIMBUCA simulation package [47]. This code allows calculation of the ion motion in a Penning trap. Furthermore, it utilizes the GPU of a graphics card in order to speed up the calculation of the ion-ion interactions in a Penning trap and thus enables the investigation of the properties of an ion cloud of more than a thousand ions. The geometry of the preparation Penning trap [25] and the applied potentials as well as realistic buffer-gas collisions have been taken into account.
All nuclides investigated in the present work decay by emission of a $\beta^{-}$particle and an anti-electron neutrino,

$$
{ }_{Z}^{A} X \longrightarrow{ }_{Z+1}^{A} Y+\beta^{-}+\overline{\nu_{e}}
$$

The daughter nucleus has maximum recoil energy when the anti-electron neutrino is at rest after decay. In this case momentum conservation requires

$$
p_{\text {recoil }}+p_{e}=0
$$

for the sum of the momenta of the recoil nucleus and the emitted electron. In the decay the energy $Q$ is released, which for $p_{\nu}=0$ is shared between the daughter nucleus and the electron

$$
E_{k i n, r e c o i l}+E_{k i n, e}=Q .
$$

Taking the relation

$$
E=E_{k i n}+m c^{2}
$$

between the total energy $E$, the kinetic energy $E_{k i n}$, and the rest mass energy $E_{0}=m c^{2}$, together with the relativistic energy-momentum relation,

$$
E^{2}=p^{2} c^{2}+m^{2} c^{4}
$$

one obtains the maximum recoil energy for the daughter nucleus in case of $\beta^{-}$decay:

$$
\begin{aligned}
E_{\text {recoil }, \text { max }} & =\frac{Q^{2}+2 E_{0, e} Q}{2\left(Q+E_{0, \text { recoil }}+E_{0, e}\right)} \\
& =\frac{Q^{2}+2 m_{e} c^{2} Q}{2\left(Q+M_{\text {recoil }} c^{2}+m_{e} c^{2}\right)} .
\end{aligned}
$$

Due to the large mass of the recoil nucleus $\left(M_{\text {recoil }} \gg\right.$ $\left.m_{e}, Q\right)$ this can be approximated by

$$
E_{\text {recoil }, \max } \approx \frac{Q\left(Q+2 m_{e} c^{2}\right)}{2 M_{\text {recoil }} c^{2}}=E_{0} .
$$

The maximum recoil energies for the cases studied in this work are listed in table 4 . Since the axial potential wall of the preparation trap is about $100 \mathrm{~V}$ at both ends, a loss of ions due to the larger recoil energy can be expected. Aside from the recoil energy, also the angle of the momentum of the recoil nucleus relative to the magnetic field axis varies. This makes trapping of recoil ions possible even if the recoil energy is higher than the trapping potential.

The distribution of recoil energies after $\beta$ decay is determined by the $\beta-\nu$ angular correlation as described in $[48,49]$. For unpolarized nuclei and with a Fierz interference coefficient $b=0$ this correlation can be written as

$$
\omega\left(\theta_{\nu \beta}\right)=1+a \frac{p_{\beta} p_{\nu}}{E_{\beta} E_{\nu}} \cos \left(\theta_{\nu \beta}\right),
$$

with the $\beta-\nu$ angular correlation coefficient $a$, the total energies $E_{\beta}$ and $E_{\nu}$ of the emitted $\beta$ particle and neutrino, respectively, and $\theta_{\beta \nu}$ the angle between the momentum 


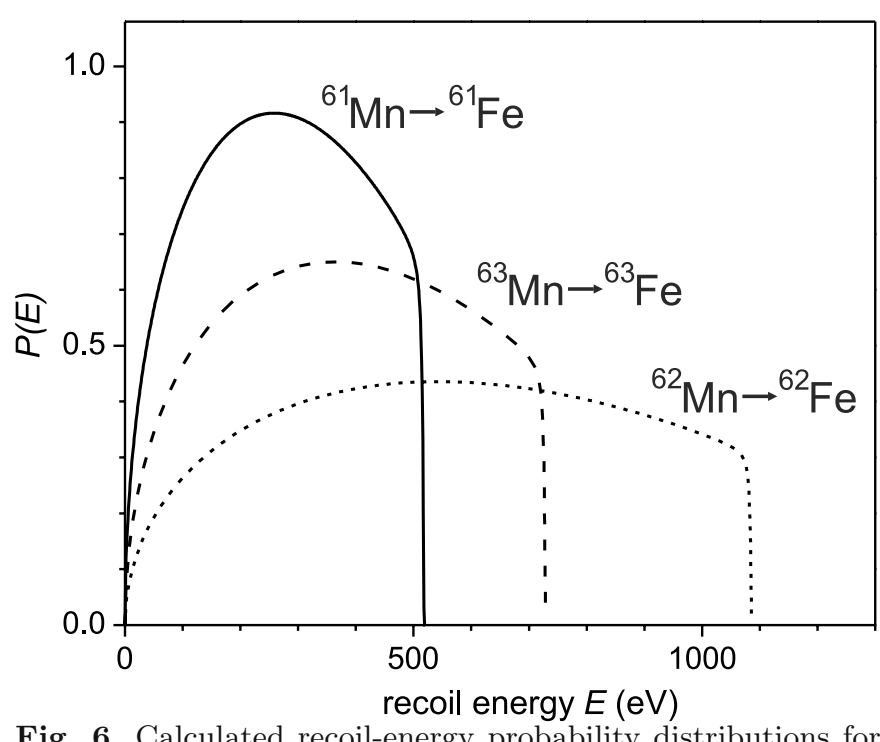

Fig. 6. Calculated recoil-energy probability distributions for the daughter nuclides after $\beta$ decay of ${ }^{61-63} \mathrm{Mn}$. Data have been normalized with respect to the area of $P(E)$.

$p_{\beta}$ and $p_{\nu}$ of the $\beta$ particle and the neutrino, respectively. For pure Fermi decays $a=1$ and for pure Gamow-Teller transitions $a=-1 / 3$. For the neutron-rich nuclides in this work only $a=-1 / 3$ is considered since Fermi transitions in their $\beta^{-}$decay are energetically forbidden and admixtures due to isospin breaking can be neglected. In order to obtain the distribution of recoil energies, eq. (9) is transformed into a distribution $P\left(E_{\text {recoil }}, E_{\beta}\right)$ as described in $[49]$, followed by an integration over all $\beta$-particle energies $E_{\beta}$. The resulting distributions $P\left(E_{\text {recoil }}\right)$ (see fig. 6$)$ are used for the simulations. The shape of the distribution is governed by the $\beta-\nu$ angular correlation coefficient $a=$ $-1 / 3$ and the only major difference between isotopes is the maximum recoil energy $E_{0}$, which is largest for ${ }^{62} \mathrm{Mn}$.

The stored manganese ions are expected to have an initial radial distribution with a FWHM of less than $2 \mathrm{~mm}$ (a Gaussian distribution is assumed). The velocity distribution of the ions is determined by their recoil energy distribution as mentioned above. Within the SIMBUCA simulation, the initial position and initial momentum (due to the recoil process) are randomly set according to the underlying probability distributions. The angle of the recoiling ion relative to the experiment axis is isotropically distributed. The magnetic field strength was set to $4.7 \mathrm{~T}$, i.e. that of the ISOLTRAP preparation Penning trap. All simulations were performed for 100000 ions with one ion in the trap in each cycle. Note that Coulomb interaction was tested in the simulation but showed no effect on the resulting ion loss for an initial distribution of ions within an FWHM of $2 \mathrm{~mm}$ and a total number of up to 500 ions stored at once. Since less than 20 ions are typically stored in each experimental cycle in the trap, the density of the ions was not high enough for Coulomb interaction to play a role and was therefore neglected in the following simulations.

The situation is different with respect to the charge state of the recoil ions. After undergoing $\beta^{-}$decay, the daughter nuclides are expected to be doubly charged un-
Eur. Phys. J. A (2012) 48: 97

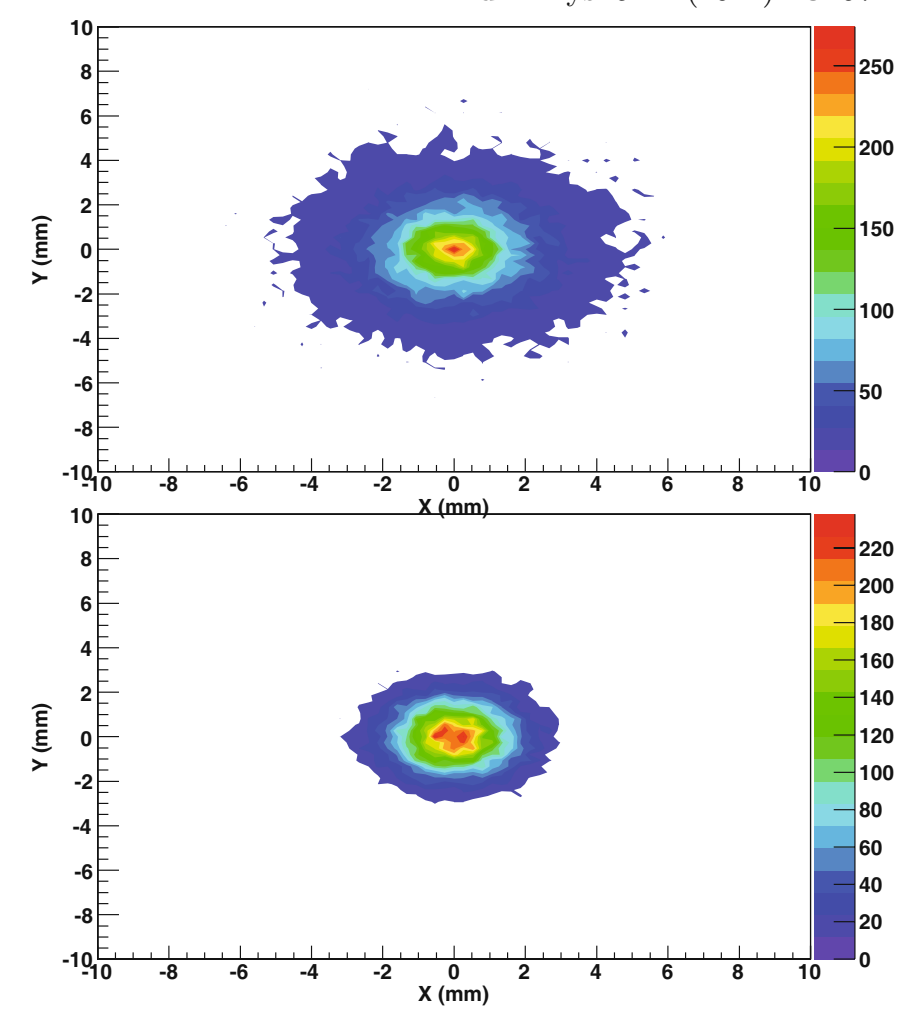

Fig. 7. (Color online) Number of recoil ions ${ }^{62} \mathrm{Fe}^{z+}$ (top: charge state $z=1$, bottom: $z=2$ ) lost in the region of the bottom electrode of the preparation Penning trap after in-trap decay of a total of $100000{ }^{61} \mathrm{Mn}^{+}$ions. The number of events is plotted as a function of the distance from the trap axis $(x=y=0)$.

less other charge-changing reactions occur, e.g., electron shake-off or charge exchange in the collision with atoms or molecules present in the trap volume. The influence of a sudden change of the charge state on the ion trajectory in a Penning trap was studied in detail in [50]. The main result of reducing the charge state from $z=2$ to $z=1$ is an increase of the radial orbit extension in the trap by a factor of up to three, which can lead to ion loss. In the present experiment, only singly charged iron ions were taken into account and their cyclotron frequency applied in the buffer-gas cooling procedure to re-center them in the preparation trap. It is assumed that the majority of daughter ions ends up in the $z=+1$ charge state. However, it is not known when the change from $z=+2$ to $z=+1$ occurs. Therefore, the simulation was performed for singly and doubly charged recoil ions, where the charge state does not change throughout the duration of the simulation of the ion trajectory, starting after $\beta$ decay until the ion is either lost or brought back to the center of the trap. Note that in the simulation the ions which hit the electrodes are lost within less than $10 \mu$ s after $\beta$ decay.

As an example, fig. 7 shows the number of ions lost in the region of the bottom electrode of the preparation trap (hitting an electrode or leaving the trapping potential) after a simulation of 100000 ions under experimental conditions. The initial parameters were a FWHM of $2 \mathrm{~mm}$ and only $\beta$ decay (no $\gamma$ decay, which will be discussed below). The top and bottom graph give the results for 


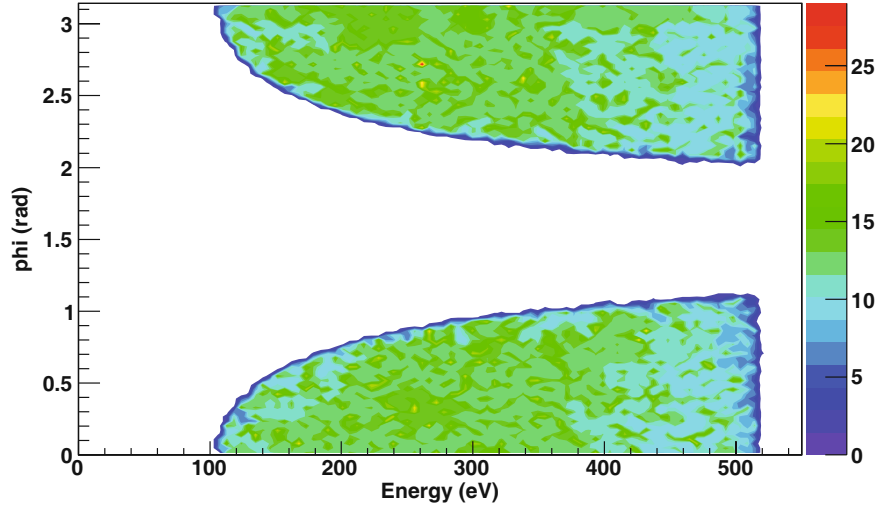

Fig. 8. (Color online) Number of ions lost as a function of the recoil energy and the angle of the momentum vector of the recoil ion directly after $\beta$ decay $(\phi=0$ and $\phi=\pi$ are in the direction of the bottom and the top of the preparation Penning trap, respectively).

singly and doubly charged recoil ions ${ }^{61} \mathrm{Fe}^{z+}$. The main ion loss occurs at small angles while the general shape of the distribution resembles a Gaussian, which is the initial ion distribution in the trap. For the doubly charged ions the distribution of ion loss is much more focused toward the experiment axis, due to the effect of the better radial storage in the homogeneous magnetic field.

In order to illustrate the influence of the recoil angle and the recoil energy on the ion loss, fig. 8 shows the outcome of another simulation: Above the threshold energy (which is deduced from the endcap voltage $100 \mathrm{~V}$ in the present case) ion loss occurs which increases for larger angles $\phi$ relative to the experiment axis. Thus, depending on the $\beta-\nu$ angular correlation coefficient $a$ the ion loss can be very different. Especially Fermi transitions $(a=1)$ favor higher recoil energies and thus ion loss is more likely to occur.

Concerning the radial ion loss, an additional simulation was performed where the FWHM of the initial ion distribution was varied. The result for the ion loss is shown in fig. 9. While for small ion clouds almost no ion loss occurs radially, it increases with increasing FWHM. The ion loss in axial direction is shown in fig. 10 as a function of the axial potential wall for ${ }^{61} \mathrm{Fe}^{1+}$. Clearly, the ion loss decreases when the potential of the trap encaps approaches the maximum recoil energy.

The fraction of ions that remain stored after in-trap decay under the different conditions are listed in table 5. As mentioned above, the ion loss for doubly-charged recoil ions is suppressed and thus a larger fraction should remain in the trap volume.

\subsection{Additional $\gamma$ decay}

After $\beta$ decay, excited energy levels of the daughter nuclide can be populated with subsequent emission of one or several gammas at energies of the order of hundreds of $\mathrm{keV}$ to several MeV. Therefore, the daughter nucleus experiences a further two-body decay event, which leads to additional

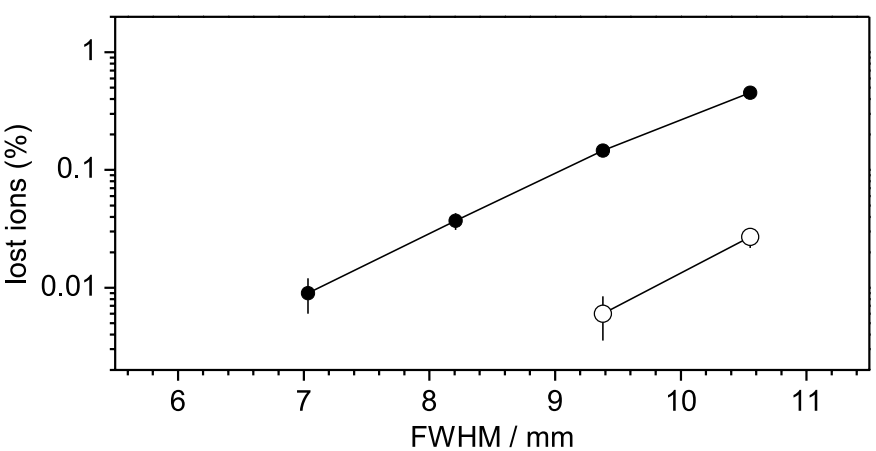

Fig. 9. Fraction of recoil ions ${ }^{61} \mathrm{Fe}^{z+}$ lost on the radial trap electrodes of the preparation Penning trap after in-trap decay as a function of the FWHM of the initial ion distribution (filled circles: $z=1$, empty circles: $z=2$ ). The potential wall was set to $100 \mathrm{~V}$ as in the experiment.

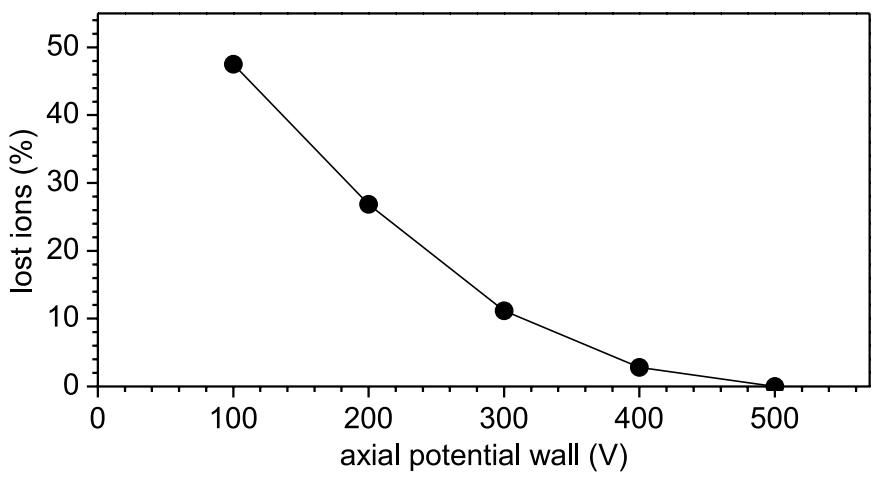

Fig. 10. Fraction of recoil ions ${ }^{61} \mathrm{Fe}^{1+}$ lost axially as a function of the potential applied to the outer Penning trap electrodes.

recoiling and a corresponding change of momentum of the daughter ion. It is assumed that the $\gamma$ decay happens instantaneously after the $\beta$ decay. This is a valid assumption since the maximum half-life of an excited state is nanoseconds while the ion has to travel more than $3 \mu$ s before it hits the wall of the Penning trap and is lost.

For the nuclides studied in this work, the level schemes are shown in fig. 11 . In the case of ${ }^{61} \mathrm{Fe}$, the $\beta$-decay feeding to the different energy levels is known $[27,51]$. In the other two cases recent experiments provided new data on the level schemes $[52,53]$ and some of the levels with strong $\beta$ feeding are marked with an arrow. If several gammas are emitted sequentially, all the recoil effects have to be combined, leading to a complicated shape of the recoil energy spectrum. Since the branching is not always well known and the uncertainty of the experimental data is too large for a precise comparison, only single- $\gamma$ emission events after $\beta$ decay are considered.

If $E^{*}$ is the excitation energy of the daughter nuclide after $\beta$ decay of the parent nuclide, energy and momentum conservation requires

$$
\begin{gathered}
E^{*}=E_{R}+E_{\gamma}, \\
0=p_{R}+p_{\gamma},
\end{gathered}
$$




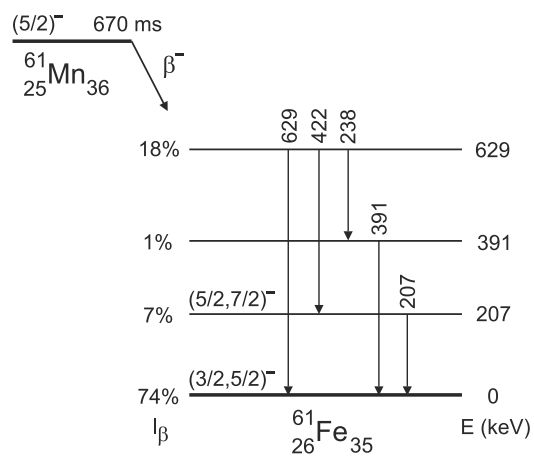

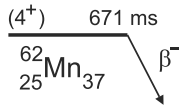

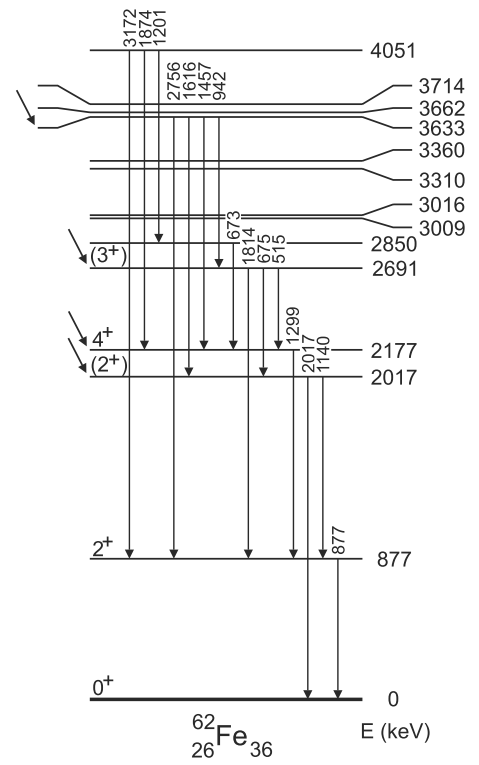

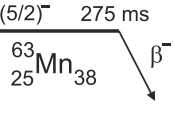
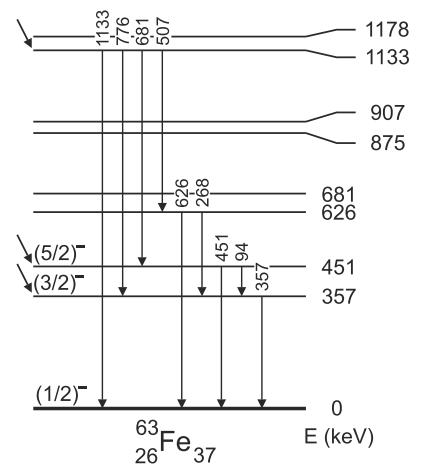

Fig. 11. Energy levels of ${ }^{61-63} \mathrm{Fe}$ populated after $\beta$ decay of ${ }^{61-63} \mathrm{Mn}$. Data from $[27,51-53]$.

with $E_{R}=\left(p_{R}\right)^{2} /(2 M)$ with $M$ the mass of the nuclide and $p_{\gamma}=E_{\gamma} / c$. The recoil energy due to emission of a $\gamma$ is then given by

$$
E_{R}=\frac{E_{\gamma}^{2}}{2 M c^{2}} .
$$

A transformation to the moving frame, i.e. the daughter nuclide after initial $\beta$ decay, can be performed using the momentum conservation

$$
\begin{aligned}
& \boldsymbol{p}_{\boldsymbol{R}}{ }^{\prime}=\boldsymbol{p}_{\boldsymbol{R}}-\boldsymbol{p}_{\boldsymbol{\gamma}}, \\
& p_{R}^{\prime 2}=p_{R}^{2}+p_{\gamma}^{2}-2 p_{R} p_{\gamma} \cos (\theta),
\end{aligned}
$$

resulting in the final recoil energy of the daughter nuclide

$$
E_{R}^{\prime}=E_{R}+\frac{E_{\gamma}^{2}}{2 M c^{2}}-\cos (\theta) \sqrt{\frac{2 E_{R} E_{\gamma}^{2}}{M c^{2}}} .
$$

In case the $\gamma$ is emitted in the direction of the moving daughter nuclide $\left(\theta=0^{\circ}\right)$ the kinetic energy is reduced. If the $\gamma$ is emitted in the opposite direction $\left(\theta=180^{\circ}\right)$ the daughter nuclide is accelerated to the maximum recoil energy. In the simulation, the angle $\theta$ is randomly distributed within the Monte Carlo approach.

Figure 12 shows the recoil-energy spectrum for ${ }^{63} \mathrm{Fe}$ where the $\gamma$ recoil is taken into account. For comparison, the dashed line gives the recoil energy spectrum without additional $\gamma$ decay, i.e. for ground-state to ground-state transitions. The dotted line shows the result for $\beta$ decay only to the excited state at $357 \mathrm{keV}$ (without the subsequent $\gamma$ decay). The solid line gives the final result including the $\gamma$ transition to the ground state. Since the $\gamma$ energy is much smaller than the $\beta$ decay energy $Q$, the effect of the additional $\gamma$ recoil can only be seen at higher recoil energies.

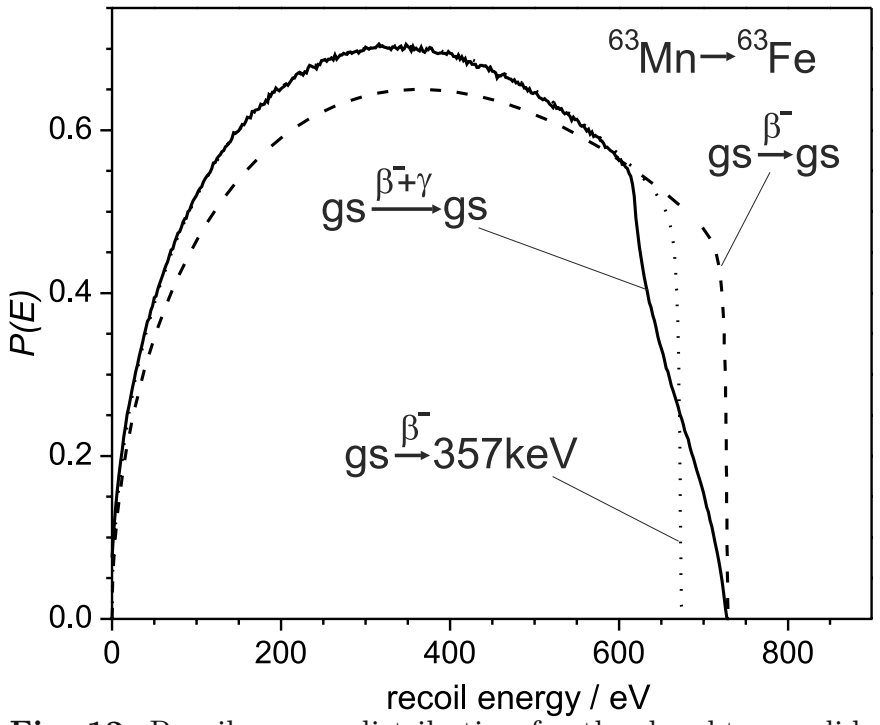

Fig. 12. Recoil energy distribution for the daughter nuclide after $\beta$ decay of ${ }^{63} \mathrm{Mn}$ to the ground state of ${ }^{63} \mathrm{Fe}$ (dashed line), to the excited state at $357 \mathrm{keV}$ (dotted line), and to the excited state at $357 \mathrm{keV}$ with subsequent $\gamma$ decay to the ground state (solid line). Data have been normalized with respect to the area of $P(E)$.

For the simulation of the in-trap decay including $\gamma$ recoil, the following approximations and considerations have been made.

i) ${ }^{61} \mathrm{Mn}$ decay: $74 \%$ of the $\beta$-decay feeding goes to the ground state of ${ }^{61} \mathrm{Fe}$, i.e. no $\gamma$ decay is involved [51]. About $7 \%$ is fed into the level at $207 \mathrm{keV}$ and about $18 \%$ into the level at $629 \mathrm{keV}$. The $\gamma$ cascades from the latter are not considered since the $629 \mathrm{keV} \gamma$ transition to the 
ground state carries most of the relative intensity, i.e. in the simulation a fraction of $18 \%$ performs a $\gamma$ decay to the ground state at the respective energy of $629 \mathrm{keV}$.

ii) ${ }^{62} \mathrm{Mn}$ decay: As recently found [52], it is the $4^{+}$state that $\beta$ decays and therefore the decay to the $0^{+}$ground state of ${ }^{62} \mathrm{Fe}$ is strongly suppressed. The most intense $\beta$ feeding goes into the $3633 \mathrm{keV}$ level, the level at $2691 \mathrm{keV}$ and the levels at $2177 \mathrm{keV}$ and $2017 \mathrm{keV}$, respectively. All but one decay proceed by $\gamma$ cascades to the $877 \mathrm{keV}$ level and from there (with the highest relative intensity) by $\gamma$ decay to the ground state. Since a complex decay scheme has to be considered, a rough estimate was performed taking into account only $\beta$-decay feeding to and subsequent $\gamma$ decay from the $877 \mathrm{keV}$ level.

iii) ${ }^{63} \mathrm{Mn}$ decay: The level scheme shown in fig. 11 is the result of a recent experiment [53] at ISOLDE/CERN and new data have been recorded but are not evaluated yet [54]. The proposed scheme $[53,54]$ shows a strong $\beta$ feeding to three levels as marked with arrows. Most of the $\gamma$ cascades end up in the $357 \mathrm{keV}$ level. In addition, the $1133 \mathrm{keV}$ level decays directly to the ground state. For the simulation $25 \%$ of $\gamma$ decay were taken from the $1133 \mathrm{keV}$ level and $75 \%$ from the $357 \mathrm{keV}$ level with no $\beta$ feeding to the ground state.

The results from these simulations are listed in table 5 . Note that the simulation was performed only for singly charged daughter nuclei. Comparing these values with the ones for the case of $\beta$ decay only, a slight increase of the surviving fraction of daughter ions can be seen. Similarly, an increase for doubly charged recoil ions can be expected.

\subsection{Comparison to experimental results}

The simulation results can now be compared to the experimental values, which are deduced from the yield measurements shown in fig. 4 . In the case of ${ }^{61} \mathrm{Mn}$ no ion loss is observed. There is, however, a large uncertainty due to fluctuations in the production at ISOLDE in the case of this experimental run (mainly a variation of the protonbeam intensity and of the RILIS ionization). Nevertheless, in agreement with the simulation results, the largest trapping efficiency is observed for ${ }^{61} \mathrm{Fe}^{+}$. For ${ }^{62} \mathrm{Fe}$ and ${ }^{63} \mathrm{Fe}$ similar experimental results are obtained with about $50 \%$ recoil-ion-trapping efficiency, which agrees with the simulation results within the uncertainties given by the fluctuations of the beam intensity. Note that only singly charged ions were observed in the experiment, i.e., the production of doubly charged daughter ions - in order to achieve better precision and higher resolving power [55]seems inefficient.

Thus, ab initio simulations confirm the experimental findings, i.e. the possibility to store daughter ions after $\beta$ decay with efficiencies of about $50 \%$. As shown by the simulations, the efficiency of the trapping depends on various experimental parameters and the parameters of the $\beta$ decay itself. First of all, the $Q$-value defines the maximum recoil energy which is directly linked to the axial potential wall of the Penning trap. In addition, the magnetic field strength $B$ influences the radial storage, i.e. radial ion loss after $\beta$ decay is more likely for smaller $B$-values. Similarly, if the ion cloud is not compressed in the trap center after injection, radial ion loss may occur. Note that the influence of the buffer-gas pressure was not studied in this work.

\section{Conclusion and outlook}

The mass excess values of neutron-rich manganese and iron isotopes have been determined by use of Penning trap mass spectrometry. Isomers have been identified for ${ }^{58} \mathrm{Mn}$ and for ${ }^{60} \mathrm{Mn}$, where the experimental results indicate that the isomeric states were more strongly populated and their mass excess values measured in the present work. In the case of ${ }^{62} \mathrm{Mn}$ the longer-lived state was more strongly populated and survived the ion preparation in the ISOLTRAP setup for mass measurement. A direct assignment of the ground state was not possible. For ${ }^{61-63} \mathrm{Mn}$ the $Q$-values were deduced from the measured masses with an absolute uncertainty of $4-7 \mathrm{keV}$.

The iron isotopes ${ }^{61-63} \mathrm{Fe}$ were produced by recoilion trapping in the buffer-gas-filled preparation trap of ISOLTRAP. The ion yields of $a b$ initio simulations agree with the values observed in the experiment, showing a storage of the recoil ions with a trapping efficiency of about $50 \%$, which is mainly influenced by the applied axial trapping potential. The simulation showed a slight reduction of ion loss if $\gamma$ decay follows the initial $\beta$ decay to an excited level.

Although the recoil-ion trapping method is limited to those nuclides which have both sufficient production yields and also half-lives in a time window between few tens of milliseconds and a few seconds (in order to obtain at least one daughter ion per cycle), it has been shown to be efficient for the present investigation. It thus allows access to nuclides which are presently otherwise not available. Furthermore, by use of the simulations reported, experimental campaigns can be optimized beforehand, i.e., by selection of storage parameters maximizing the yield of daughter nuclei.

This work was supported by the German BMBF under contracts 06MZ215, 06GF186I, and 06GF9102, by the EU within the 6th Framework through RII3-EURONS (contract no. 506065) and Marie Curie Fellowship MEIF-CT-2006-042114, by the Helmholtz association of national research centers (HGF) under contract VH-NG-037, by the French IN2P3, and by the Max-Planck Society. RBC acknowledges support from Turkish Atomic Energy Authority (TAEK) 04K120100-4 and the Humboldt Foundation. We also thank Luis Fraile, Karsten Riisager, and William Walters for fruitful discussions.

\section{References}

1. N. Aoi et al., Phys. Rev. Lett. 102, 012502 (2009).

2. A. Gade et al., Phys. Rev. C 81, 051304 (2010).

3. O. Sorlin et al., Eur. Phys. J. A 16, 55 (2003).

4. P. Adrich et al., Phys. Rev. C 77, 054306 (2008).

5. D. Pauwels et al., Phys. Rev. C 79, 044309 (2009). 
6. W. Rother et al., Phys. Rev. Lett. 106, 022502 (2011).

7. M. Hannawald et al., Phys. Rev. Lett. 82, 1391 (1999).

8. J. Ljungvall et al., Phys. Rev. C 81, 061301 (2010).

9. K. Wimmer et al., Phys. Rev. Lett. 105, 252501 (2010).

10. C. Guénaut et al., Phys. Rev. C 75, 044303 (2007).

11. K. Blaum, Phys. Rep. 425, 1 (2006).

12. L. Schweikhard, G. Bollen (Editors), Ultra-accurate mass spectometry and related topics, in Int. J. Mass Spectrom., Vol. 251, issues 2-3 (2006).

13. G. Savard et al., Hyperfine Interact. 132, 223 (2001).

14. J. Dilling et al., Int. J. Mass Spectrom. 251, 198 (2006).

15. M. Block et al., Eur. Phys. J. D 45, 39 (2007).

16. J. Ketelaer et al., Nucl. Instrum. Methods A 594, 162 (2008).

17. V.S. Kolhinen et al., Nucl. Instrum. Methods A 600, 391 (2009).

18. R. Ringle et al., Nucl. Instrum. Methods A 604, 536 (2009).

19. T. Eronen et al., Eur. Phys. J. A 48, 46 (2012).

20. E. Kugler, Hyperfine Interact. 129, 23 (2000).

21. M. Mukherjee et al., Eur. Phys. J. A 35, 1 (2008).

22. A. Herlert et al., New J. Phys. 7, 44 (2005).

23. V.I. Mishin et al., Nucl. Instrum. Methods B 73, 550 (1993).

24. F. Herfurth et al., Nucl. Instrum. Methods A 469, 254 (2001).

25. H. Raimbault-Hartmann et al., Nucl. Instrum. Methods B 126, 378 (1997).

26. G. Savard et al., Phys. Lett. A 158, 247 (1991).

27. ENSDF database (Evaluated Nuclear Structure Data File), http://www.nndc.bnl.gov/ensdf/.

28. G. Audi, O. Bersillon, J. Blachot, A.H. Wapstra, Nucl. Phys. A 729, 3 (2003).

29. A. Herlert et al., Int. J. Mass Spectrom. 251, 131 (2006).

30. M. König et al., Int. J. Mass Spectrom. Ion Processes 142, 95 (1995).
31. A.H. Wapstra, G. Audi, C. Thibault, Nucl. Phys. A 729, 129 (2003).

32. G. Audi, A.H. Wapstra, C. Thibault, Nucl. Phys. A 729, 337 (2003).

33. A. Kellerbauer et al., Eur. Phys. J. D 22, 53 (2003).

34. I.F. Croall, H.H. Willis, J. Inorg. Nucl. Chem. 24, 221 (1962).

35. U. Köster et al., Nucl. Instrum. Methods B 204, 347 (2003).

36. U. Köster, CERN-THESIS-2001-002, PhD thesis, TU München, Germany (1999).

37. S. Naimi et al., Phys. Rev. C 86, 014325 (2012) doi:10.1103/PhysRevC.86.014325.

38. C. Guénaut, private communication.

39. C. Guénaut et al., Eur. Phys. J. A 25, s01, 35 (2005) doi:101140/epjad/i2005-06-030-4.

40. S.N. Liddick et al., Phys. Rev. C 73, 044322 (2006).

41. L. Gaudefroy et al., Eur. Phys. J. A 23, 41 (2005).

42. M. Oinonen et al., Hyperfine Interact. 127, 431 (2000).

43. M. Block et al., Phys. Rev. Lett. 100, 132501 (2008).

44. T. Eronen et al., Phys. Rev. Lett. 103, 252501 (2009).

45. S. Eliseev et al., Phys. Rev. Lett. 106, 052504 (2011).

46. D. Fink et al., Phys. Rev. Lett. 108, 062502 (2012).

47. S. Van Gorp et al., Nucl. Instrum. Methods A 638, 192 (2011).

48. N. Severijns, M. Beck, O. Naviliat-Cuncic, Rev. Mod. Phys. 78, 991 (2006).

49. O. Kofoed-Hansen, Dan. Mat. Fys. Medd. 28, 1 (1954).

50. A. Herlert, L. Schweikhard, Int. J. Mass Spectrom. 234, $161(2004)$.

51. E. Runte et al., Nucl. Phys. A 441, 237 (1985).

52. N. Hoteling et al., Phys. Rev. C 82, 044305 (2010).

53. H. Mach et al., Acta. Phys. Pol. 40, 477 (2009).

54. L.M. Faile, private communication.

55. A.T. Gallant et al., Phys. Rev. C 85, 044311 (2012). 